\title{
Bipolar and Schizophrenia Disorders Diagnosis Using Artificial Neural Network
}

\author{
Mateus Beck Fonseca1,2* (D), Renan Soares de Andrades', Suelen de Lima Bach², \\ Carolina David Wiener ${ }^{2,3}$, Jean Pierre Oses ${ }^{2,4}$ \\ ${ }^{1}$ Engineer Center, Federal University of Pelotas, Pelotas, Brazil \\ ${ }^{2}$ Department of Health and Behavior, Clinical Neuroscience Lab, Catholic University of Pelotas, Pelotas, Brazil \\ ${ }^{3}$ Anhanguera Educacional, Rio Grande, Brazil \\ ${ }^{4}$ Department of Electronic Engineering and Computer, Catholic University of Pelotas, Pelotas, Brazil \\ Email: ^mateus.fonseca@ufpel.edu.br
}

How to cite this paper: Fonseca, M.B., de Andrades, R.S., de Lima Bach, S., Wiener, C.D. and Oses, J.P. (2018) Bipolar and Schizophrenia Disorders Diagnosis Using Artificial Neural Network. Neuroscience \& Medicine, 9, 209-220.

https://doi.org/10.4236/nm.2018.94021

Received: November 29, 2018

Accepted: December 24, 2018

Published: December 27, 2018

Copyright $\odot 2018$ by authors and Scientific Research Publishing Inc. This work is licensed under the Creative Commons Attribution International License (CC BY 4.0).

http://creativecommons.org/licenses/by/4.0/

\begin{abstract}
Motivation: Bipolar disorder (BD) and schizophrenia (SZ) has a difficult diagnosis, so the main objective of this article is to propose the use of Artificial Neural Networks (ANNs) to classify (diagnose) groups of patients with BD or SZ from a control group using sociodemographic and biochemical variables. Methods: Artificial neural networks are used as classifying tool. The data from this study were obtained from the array collection from Stanley Neuropathology Consortium databank. Inflammatory markers and characteristics of the sampled population were the inputs variables. Results: Our findings suggest that an artificial neural network could be trained with more than $90 \%$ accuracy, aiming the classification and diagnosis of bipolar, schizophrenia and control healthy group. Conclusion: Trained ANNs could be used to improve diagnosis in Schizophrenia and Bipolar disorders.
\end{abstract}

\section{Keywords}

Bipolar Disorder, Schizophrenia Disorder, Biomarkers, Artificial Neural Network

\section{Introduction}

Artificial neural network (ANN) attempts to use multiple layers of calculations to emulate the neuronal circuitry in the human brain by interpreting and drawing conclusions from several information. ANN algorithms are mathematical models based on biological neural systems that simulate the behavior of neurons. The neuron receives inputs from multiple neurons and outputs a value based 
upon the activation function [1]. The ANN inputs are multiplied by different weights to generate a predictive response. So, these responses in ANN are widely used for several applications such as classification and pattern recognition [2]. This tool is effective modeling non-linear relationships that may be a promising candidate for differentiation for several biological processes [3]. ANN are used in medical field to analysis of sleep disorders, cytopathology and histopathology such as classification of breast cancer images and others, in prediction of heart disease, CD4+ T cell differentiation and immune cell subset classification, combining clinical predictors of antidepressant response in mood disorder, and other classifications [4] [5].

Bipolar disorder (BD) and schizophrenia (SZ) are complex mental disorders with high genetic load, and are the largest global contributors to years with functional disability [6]. These disorders are among the most severe psychiatric disorders that affects around $1 \%$ and $0.8 \%$ of the general population, respectively [7] [8] [9]. BD is characterized by depressive, (hypo)manic, or mixed episodes [10]. SZ is characterized by amotivational, disorganized, affective, delusional, hallucinatory, or catatonic symptoms [10]. Both have been associated with negative health outcomes and progressive impairments [11] [12]. Therefore improve the diagnosis may be associated with a better definition of the treatment and lower damages to subject. Several evidences point out that psychiatric disorders have several changes in the molecular and functional mechanisms of the neuron, leading to observable changes in the brain [13]. Studies suggest that stressful events are important in the early stages of the disease [14], so the phenotypic manifestation of mood disorders is presumably the result of the interaction between the effects of environmental stress and genetic predisposition.

$\mathrm{BD}$ and $\mathrm{SZ}$ have been associated with alteration in inflammatory cytokine levels, including Interleukin (IL-1, IL-6, IL-18, and IL-10), tumor necrosis factor alpha (TNF- $\alpha$ ) and beta (TNF- $\beta$ ), transforming growth factor beta (TGF- $\beta$ ), and interferon gamma (IFN $\gamma$ ), when compared to healthy controls, and has been associated to neurotrophic factors changes [15] [16] [17] [18] [19]. These cytokines are produced by a variety of cell types including immune cells, muscular cells, glial cells and neurons; they mediate signaling between immune cells, and are mainly secreted from monocytes, macrophages or lymphocytes [20]. Moreover, cytokines play a central role in the control and modulation of inflammatory responses, and modulate the neurotrophins, with a constant balance between proinflammatory and anti-inflammatory cytokines [21].

In the last years, there has been an upsurge of interest within the neuroscience community in the use of artificial intelligence (AI) methods, including ANN [22] [23]. Moreover, ANN analyses are gaining traction in psychiatric research, providing predictive models for both clinical practice and public health systems. Compared with traditional statistical methods that provide primarily average group-level results, machine-learning algorithms provide predictions and stratification of clinical outcomes at the level of an individual subject [24]. However, for the best of our knowledge there is no ANN using an accessible peripheral 
biomarker (inflammatory interleukins) to classify the outcome in BP and SZ patients. In this way, the main objective of this article is to propose the use of ANN to classify (diagnose) groups of patients with BP or SZ from a control group.

\section{Methods}

\subsection{Participants}

The data from this study were obtained from the Array collection from Stanley Neuropathology Consortium databank (SNC) [25] [26]. The databank consists of 105 brains samples ( 35 schizophrenia brains, 35 bipolar disorder brains, and 35 health control brains). For one trainingscenario, schizophrenia and bipolar disorder were grouped as outcome to perform the analysis, so 35 samples were randomly drawn to match the control group. As input variables, 34 sociodemographic and biochemical variables were applied to train an ANN. The ANN training data input variables were: age, sex, smoking, BDNF, IFN-gamma, IgA, IgE, IgM, IL-1alpha, IL-1beta, IL-1ra, IL-2, IL-3, IL-5, IL-6, IL-6 Receptor, IL-7, IL-8, IL-10, IL-11, IL-12p40, IL-12p70, IL-13, IL-15, IL-16, IL-17, IL-17E, IL-18, IL-23, NGFb, RANTES, TGF-alpha, TNF RII, TNF-alpha, TNF-beta.

\subsubsection{Selection, Clinical Information, and Diagnosis}

Briefly, donors for the brain collection are identified by investigators in original study. Individuals over age 65 are excluded because of the increased likelihood of comorbid neurological disorders. A preliminary diagnosis and requests permission for donation of the brain and for release of the deceased's medical records was solicited. Data regarding the sociodemographic, clinical and psychiatric history, substances use was collected. Medical and psychiatric records are requested for known hospitalizations and outpatient treatments to be made until sufficient information has been collected to make a clear diagnosis. All records was reviewed by one psychiatrist and the information is entered into a computerized database (demographic data, family history, education, age of onset, total duration of hospitalizations, psychiatric diagnosis, cause of death, medical diagnoses, medications at time of death, brain weight, interval between death and refrigeration of body, and interval between death and freezing of brain tissue [postmortem interval (PMI)]) by identifying number only (more details see [25]). After all information was collected, the DSM-IV [10] psychiatric diagnosis was made independently by two senior psychiatrists. If there was disagreement between them, the records were made by a third senior psychiatrist.

\subsubsection{Processing of Brain Tissue}

Trained medical examiners collected and processed the brain tissue. Half of brain was fixed in formalin while the other is cut into $1.5 \mathrm{~cm}$ thick coronal slices and frozen in a mixture of isopentane and dry ice. The frozen half was stored at $-70^{\circ} \mathrm{C}$ until analysis.

\subsubsection{Neuropathology Consortium}

The Stanley Medical Research Institute (SMRI) [25] provides postmortem brain 
tissue for research since 1994 facilitating the number and quality of neuropathology studies for the major psychiatric disorders and to identify possible targets for drug development. In this context, the arraycollection [26] provides samples with 35 cases in each of three groups: SZ, BD and unaffected controls. The diagnostic groups in collections are matched for the descriptive variables, age, gender, race, postmortem interval, mRNA quality (RIN), brain $\mathrm{pH}$ and hemisphere [25] [27]. All samples were collected between September 1994 and February 1997. The specimens that constitute the Neuropathology Consortium are made available without charge to research groups around the world.

\subsubsection{Multiplex Immunoassay Analysis}

All analytes were measured by multiplex immunoassay. Extracts $(200 \mu \mathrm{L})$ were analyzed using the Discovery MAP $^{\mathrm{TN}}$ multiplexed immunoassay panel at $\mathrm{My}$ riad-RBM (Austin, TX, USA). Each assay was calibrated using duplicate 8-point standard curves, and raw intensity measurements were interpreted into final protein concentrations using proprietary software. Machine performance was verified using quality control samples at low, medium, and high levels for each analyte [28].

\subsection{ANN Training and Statistical Analyses}

In this study the inputs to the first layer of the neural network consist of 34 sociodemographic and biochemical variables while the target output consist of the following outputs trainings classifications: 1) control or case group; 2) control or BD group; 3) control or SZ group; and 4) BD or SZ group. The network is then trained to attempt to predict response from the set of variables. Supervised ANNs were applied in this work. Supervised ANNs means that the output is already know, in a training data bank. Supervised ANNs calculate an error function between the desired fixed output (target) and their own output, and adjust the connection strengths (weights) during the training process to minimize the result of the error function. The trained ANN can be seen as an equation, which translate the ANNs inputs into outputs, and rules by which the weights are modified to minimize the error of the equation [29]. A general ANN can be identified in Figure 1, this topology has $p$ inputs, one weight connected in each input, $k$ neurons in parallel in a Hidden (or middle) layer, with a non-linear activation function, and one neuron in output layer, with linear activation function, for one output variable. This model of ANN can approximate the output of any continuous function [2], and was used in this work to classify the diagnosis.

To perform ANN training analyses, the OpenNN software was used. It is a multiplatform and open source software, for artificial neural networks [30]. In this work, the weights were randomized at the start of each training, and trained until the performance increase was above 1e- 6 with the quasi-newton method. Also, the data bank was divided for training and testing, being $80 \%$ for training and $20 \%$ of the bank for test. The ANN training gives as result a confusion matrix. The accuracy, sensitivity, specificity and F1 score (harmonic mean between 


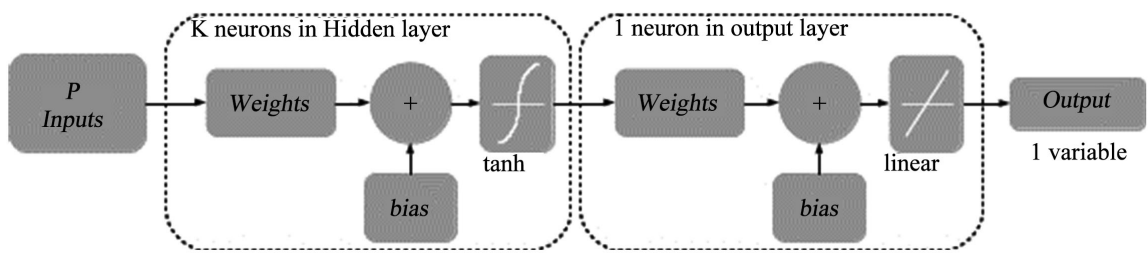

Figure 1. Neural network representation with $\mathrm{p}$ inputs, $\mathrm{k}$ neurons in hidden (middle) layer and one output neuron for one output variable. Note that is possible to have $\mathrm{p} \neq \mathrm{k}$, provided that each input has its weights. In this work we used 35 inputs (brain inflammatory markers and characteristics of the sampled population) and the output is binary as a classification of control group or BD or SZ disease. We vary the neurons in hidden layer to compare results and found the best suitable network.

precision and sensitivity) were obtained from confusion matrix calculation. Some related works also show classification functions to analyze the ANN training [31] [32]. In this work, we performed ten trainings for each number of neurons in hidden layer. They are show as mean and standard deviation (mean \pm S.D.) and the best achieved result of these trainings is also presented.

Statistical analyzes were performed by the Statistical Program for Social Sciences (SPSS) 22.0. The Chi Square and Analysis of Variance (ANOVA) test were used to check the consistency of match between groups.

\section{Results}

The analysis was conducted with 104 data samples. The original data bank was composed of 105 individuals, 35 in bipolar disorder, 35 in schizophrenia and 35 in control group. One case of bipolar disorder has no data available, so training and analysis with $\mathrm{BD}$ were matched to 34 individuals.

The characteristics of patients are shown in Table 1. In BD group, most subjects were female (67\%) while in SZ and control groups were male (74\% in both groups) ( $\mathrm{p}=0.025) .44 \%$ of $\mathrm{BD}$ patients and $20 \%$ of SZ patients are suicide victims, although there are no cases in control group ( $\leq 0.001)$. The mean age was $45.4 \pm 10.67,42.57 \pm 8.47$, and $44.2 \pm 7.58$ years for $\mathrm{BD}, \mathrm{SZ}$ and control respectively. The duration of illness was $20.00 \pm 9.62$ and $21.29 \pm 10.14$ years for BD and SZ, respectively. In addition, the lifetime antipsychotics were $1.13 \pm 2.62$ and $9.70 \pm 11.45$ years for BD and SZ, respectively. Smoking data was also available, but due to the more than forty percent of missing values, these data was not take intoaccount at the training or in Table 1. For the duration of illness, lifetime antipsychotics and suicide status, there were no event in control group, so these variables were not used in ANN training. Demographic data and complementary information were provided in Stanley Foundation research site [33].

ANN training results changing the comparisons groups can be analyzed in Tables 2-5. They present the best result of ten different training and the mean and \pm standard deviation of these trainings. The classification training results can be seen as accuracy, F1 score, sensitivity and specificity in these tables. For the best results, the displayed data stands for the best accuracy case, taking the F1 
score, sensitivity and specificity of this best case, using F1 score as tiebreaker when needed. ANN middle layer neurons were also compared from three to nine neurons. For less than three and more than nine neurons in ANN hidden layer, the accuracy results was below 50\% (data not show).

A classification with cases and controls were performed. BD and SZ were grouped, (samples were randomly drawn to match the control group), in one patient cases group. An ANN training was done to classify cases and control groups. Results of these training can be seen in Table 2. Results in Table 2 show the best achieved accuracy, F1 score, sensitivity and specificity; they were respectively $0.93,0.95,1.00$ and 0.80 for three neurons.

For differentiation of $\mathrm{BD}$ from healthy people, $\mathrm{ANN}$ training results can be seen in Table 3. One sample from control group was randomly drawn to match the $\mathrm{BD}$ group data sample. In Table 3 best training result is for six neurons, using F1 score as tiebreaker. This gives 0.92 of accuracy, 0.95 of F1 score, 1.00 for sensitivity and 0.75 for specificity. So with six neurons in ANN hidden layer, $92 \%$ of accuracy diagnosis can be achieved, recognizing all BD patients.

Schizophrenia patients group is differentiated from healthy group in Table 4. Table 4 best results for accuracy, F1 score, sensitivity and specificity, were respectively $0.93,0.93,1.00,0.86$ for seven neurons in ANN hidden layer. So, one can classify with 93 of accuracy a SZ patient from healthy people, correctly identifying all SZ patients.

Table 5 shows BD and SZ ANN training classification results. One sample from $\mathrm{SZ}$ group was randomly drawn to match the $\mathrm{BD}$ group data sample. It exemplifies classifications where the $\mathrm{BD}$ and SZ diagnosis is not clear. With three neurons in ANN hidden layer, the best result of training is achieved, and is 0.92 of accuracy, 0.93 of F1 score, 0.88 of sensitivity and 1.00 of specificity. This leads to a diagnosis of $92 \%$ of accuracy, with all SZ patients correctly identified.

Table 1. Characteristics of the sampled population for cases and control group.

\begin{tabular}{|c|c|c|c|c|}
\hline Variables & $\mathrm{BD}$ & SZ & CTRL & p-value \\
\hline & $(\mathrm{n}=34)$ & $(n=35)$ & $(\mathrm{n}=35)$ & \\
\hline Gender $^{\mathrm{a}}$ & & & & 0.025 \\
\hline Male & $16(39)$ & $26(74)$ & $26(74)$ & \\
\hline Female & $18(67)$ & $9(26)$ & $9(26)$ & \\
\hline Suicide Status ${ }^{\mathrm{a}}$ & $15(44)$ & $7(20)$ & - & $\leq 0.001$ \\
\hline Age $^{\mathrm{b}}$ & $45.40 \pm 10.67$ & $42.57 \pm 8.47$ & $44.20 \pm 7.58$ & 0.422 \\
\hline PMI Pos Morten Interval (h) ${ }^{b}$ & $37.90 \pm 18.62$ & $31.40 \pm 15.54$ & $29.37 \pm 12.87$ & 0.071 \\
\hline Brain $\mathrm{PH}^{\mathrm{b}}$ & $6.43 \pm 0.30$ & $6.47 \pm 0.24$ & $6.60 \pm 0.27$ & 0.018 \\
\hline Duration of Illness (years) ${ }^{\mathrm{b}}$ & $20.00 \pm 9.62$ & $21.29 \pm 10.14$ & - & $\leq 0.001$ \\
\hline Lifetime Antipsychotics (years) ${ }^{\mathrm{b}, \mathrm{c}}$ & $1.13 \pm 2.62$ & $9.70 \pm 11.45$ & - & $\leq 0.001$ \\
\hline
\end{tabular}

$\mathrm{BD}=$ Bipolar disorder, $\mathrm{SZ}=$ schizophrenia, and CTRL $=$ control. ${ }^{\mathrm{a}}$ Simple and relative frequencies (\%),

${ }^{\mathrm{b}}$ Mean and standard deviation, ${ }^{\mathrm{c}} 12$ cases in BD with no use of Antipsychotics, while all cases in SZ used. 
Table 2. Cases (BD and SZ grouped) and control groups ANN classification training results.

\begin{tabular}{ccccccccc}
\hline & \multicolumn{2}{c}{ Accuracy } & \multicolumn{2}{c}{ F1 Score } & \multicolumn{2}{c}{ Sensitivity } & \multicolumn{2}{c}{ Specificity } \\
\hline \#Neurons & Best result & Mean \pm S.D. & Best result & Mean \pm S.D. & Best result & Mean \pm S.D. & Best result & Mean \pm S.D. \\
\hline 3 & 0.93 & $0.66 \pm 0.12$ & 0.95 & $0.64 \pm 0.16$ & 1.00 & $0.68 \pm 0.21$ & 0.80 & $0.62 \pm 0.26$ \\
4 & 0.79 & $0.62 \pm 0.08$ & 0.80 & $0.59 \pm 0.16$ & 0.75 & $0.58 \pm 0.20$ & 0.83 & $0.62 \pm 0.23$ \\
5 & 0.71 & $0.63 \pm 0.08$ & 0.78 & $0.63 \pm 0.12$ & 0.91 & $0.63 \pm 0.20$ & 1.00 & $0.60 \pm 0.19$ \\
6 & 0.79 & $0.64 \pm 0.07$ & 0.80 & $0.58 \pm 0.12$ & 0.86 & $0.57 \pm 0.18$ & 0.71 & $0.71 \pm 0.11$ \\
7 & 0.93 & $0.68 \pm 0.12$ & 0.94 & $0.67 \pm 0.14$ & 1.00 & $0.67 \pm 0.19$ & 0.83 & $0.65 \pm 0.18$ \\
8 & 0.86 & $0.65 \pm 0.10$ & 0.80 & $0.63 \pm 0.14$ & 0.86 & $0.63 \pm 0.14$ & 0.89 & $0.60 \pm 0.33$ \\
9 & 0.79 & $0.63 \pm 0.09$ & 0.77 & $0.58 \pm 0.22$ & 0.83 & $0.57 \pm 0.23$ & 0.75 & $0.65 \pm 0.20$ \\
\hline
\end{tabular}

Result for each number of Neurons and results shown for 10 different ANN training for each number of neurons in hidden layer (70 different trainings). Accuracy, F1 Score, Sensitivity and Specificity, are presented as mean and standard deviation (S.D.).

Table 3. Bipolar disorder and control groups ANN classification training results.

\begin{tabular}{ccccccccc}
\hline & \multicolumn{2}{c}{ Accuracy } & \multicolumn{2}{c}{ F1 Score } & \multicolumn{2}{c}{ Sensitivity } & \multicolumn{2}{c}{ Specificity } \\
\hline \#Neurons & Best result & Mean \pm S.D. & Best result & Mean \pm S.D. & Best result & Mean \pm S.D. & Best result & Mean \pm S.D. \\
\hline 3 & 0.77 & $0.55 \pm 0.05$ & 0.82 & $0.57 \pm 0.08$ & 0.78 & $0.59 \pm 0.16$ & 0.75 & $0.56 \pm 0.19$ \\
4 & 0.69 & $0.56 \pm 0.05$ & 0.75 & $0.58 \pm 0.10$ & 1.00 & $0.62 \pm 0.19$ & 0.43 & $0.48 \pm 0.20$ \\
5 & 0.92 & $0.65 \pm 0.12$ & 0.94 & $0.64 \pm 0.11$ & 0.89 & $0.67 \pm 0.15$ & 1.00 & $0.65 \pm 0.25$ \\
6 & 0.92 & $0.60 \pm 0.12$ & 0.95 & $0.58 \pm 0.22$ & 1.00 & $0.64 \pm 0.28$ & 0.75 & $0.51 \pm 0.23$ \\
7 & 0.85 & $0.59 \pm 0.09$ & 0.80 & $0.58 \pm 0.09$ & 0.67 & $0.59 \pm 0.13$ & 1.00 & $0.61 \pm 0.15$ \\
8 & 0.77 & $0.55 \pm 0.09$ & 0.80 & $0.58 \pm 0.11$ & 0.86 & $0.69 \pm 0.19$ & 0.67 & $0.43 \pm 0.15$ \\
9 & 0.77 & $0.56 \pm 0.10$ & 0.77 & $0.56 \pm 0.15$ & 0.83 & $0.58 \pm 0.17$ & 0.71 & $0.54 \pm 0.19$ \\
\hline
\end{tabular}

Results shown for 10 different ANN training for each number of neurons in hidden layer (70 different trainings). Accuracy, F1 Score, Sensitivity and Specificity, are presented as mean and standard deviation (S.D.), and the best result for each number of Neurons.

Table 4. Schizophrenia and control groups ANN classification training results.

\begin{tabular}{cccccccccc}
\hline & \multicolumn{2}{c}{ Accuracy } & \multicolumn{2}{c}{ F1 Score } & \multicolumn{2}{c}{ Sensitivity } & \multicolumn{2}{c}{ Specificity } \\
\hline \#Neurons & Best result & Mean \pm S.D. & Best result & Mean \pm S.D. & Best result & Mean \pm S.D. & Best result & Mean \pm S.D. \\
\hline 3 & 0.79 & $0.57 \pm 0.09$ & 0.82 & $0.57 \pm 0.14$ & 1.00 & $0.59 \pm 0.21$ & 0.57 & $0.57 \pm 0.20$ \\
4 & 0.71 & $0.55 \pm 0.07$ & 0.71 & $0.56 \pm 0.09$ & 0.63 & $0.54 \pm 0.14$ & 0.83 & $0.58 \pm 0.16$ \\
5 & 0.71 & $0.51 \pm 0.08$ & 0.71 & $0.54 \pm 0.15$ & 0.71 & $0.57 \pm 0.21$ & 0.71 & $0.43 \pm 0.25$ \\
6 & 0.71 & $0.51 \pm 0.08$ & 0.67 & $0.50 \pm 0.09$ & 0.67 & $0.52 \pm 0.16$ & 0.75 & $0.52 \pm 0.17$ \\
7 & 0.93 & $0.56 \pm 0.13$ & 0.93 & $0.52 \pm 0.17$ & 1.00 & $0.48 \pm 0.22$ & 0.86 & $0.66 \pm 0.15$ \\
8 & 0.86 & $0.55 \pm 0.14$ & 0.86 & $0.49 \pm 0.20$ & 0.75 & $0.51 \pm 0.27$ & 1.00 & $0.60 \pm 0.13$ \\
9 & 0.79 & $0.54 \pm 0.11$ & 0.77 & $0.54 \pm 0.12$ & 0.83 & $0.60 \pm 0.24$ & 0.75 & $0.51 \pm 0.20$ \\
\hline
\end{tabular}

Results shown for 10 different ANN training for each number of neurons in hidden layer (70 different trainings). Accuracy, F1 Score, Sensitivity and Specificity, are presented as mean and standard deviation (S.D.), and the best result for each number of Neurons. 
Table 5. Bipolar disorder and Schizophrenia ANN classification training results.

\begin{tabular}{ccccccccc}
\hline & \multicolumn{3}{c}{ Accuracy } & & F1 Score & \multicolumn{2}{c}{ Sensitivity } & \multicolumn{2}{c}{ Specificity } \\
\hline \#Neurons & Best result & Mean \pm S.D. & Best result & Mean \pm S.D. & Best result & Mean \pm S.D. & Best result & Mean \pm S.D. \\
\hline 3 & 0.92 & $0.53 \pm 0.12$ & 0.93 & $0.81 \pm 0.30$ & 0.88 & $0.58 \pm 0.17$ & 1.00 & $0.56 \pm 0.23$ \\
4 & 0.77 & $0.57 \pm 0.08$ & 0.80 & $0.59 \pm 0.12$ & 0.86 & $0.64 \pm 0.16$ & 0.67 & $0.48 \pm 0.10$ \\
5 & 0.92 & $0.57 \pm 0.11$ & 0.91 & $0.59 \pm 0.12$ & 1.00 & $0.62 \pm 0.17$ & 0.88 & $0.59 \pm 0.12$ \\
6 & 0.77 & $0.54 \pm 0.10$ & 0.84 & $0.53 \pm 0.15$ & 0.89 & $0.55 \pm 0.19$ & 0.50 & $0.50 \pm 0.14$ \\
7 & 0.62 & $0.52 \pm 0.04$ & 0.74 & $0.52 \pm 0.10$ & 1.00 & $0.61 \pm 0.23$ & 0.17 & $0.45 \pm 0.31$ \\
8 & 0.77 & $0.58 \pm 0.09$ & 0.80 & $0.60 \pm 0.09$ & 1.00 & $0.65 \pm 0.13$ & 0.57 & $0.50 \pm 0.17$ \\
9 & 0.77 & $0.57 \pm 0.09$ & 0.84 & $0.60 \pm 0.08$ & 0.80 & $0.62 \pm 0.12$ & 0.67 & $0.53 \pm 0.16$ \\
\hline
\end{tabular}

Results shown for 10 different ANN training for each number of neurons in hidden layer (70 different trainings). Accuracy, F1 Score, Sensitivity and Specificity, are presented as mean and standard deviation (S.D.), and the best result for each number of Neurons.

\section{Discussion}

In present study was applied an ANN to discriminate two mental disorders and health subjects in clinical and inflammatory-based data. The ANN model was able to identify correctly a high percentage of subjects with a psychiatric disorder in a sample with sick and healthy individuals. Moreover, the ANN model shows very specific and sensitive, in confusion matrix interpretation. In our knowledge, this is the first study that proposes an ANN model to improve the use of markers and clinical data in diagnoses of BD and SZ. Our analyses suggest that an ANN function could properly classify the cases and control groups of these disorders.

Studies investigating the impact of a variety of inflammatory stimuli on the brain and behavior have reported evidence that inflammation and the release of inflammatory cytokines affect the relevant circuits for BD and SZ [16] [28]. Inflammatory cytokines reach the brain and are associated with increased expression of pro-inflammatory eicosanoids, nitric oxide, TNF- $\alpha$, IL- $1 \beta$, reactive oxygen species, as well as monocytes and macrophages in the brain [17]. Several studies have suggested an imbalance in pro and anti-inflammatory responses in the pathogenesis of $\mathrm{SZ}$ and $\mathrm{BD}$. However, the mechanisms involved in these processes remain unknown. Thus, the results found in our study agree with previous studies where there is an involvement of the immune system in these disorders. There is clinical evidence that mood disorders are immunoinflammatory disorders characterized, among other things, by the increase of proinflammatory cytokines [16] [17]. These evidences have stimulated the search for relevant peripheral markers, and there are several indications of relationships between metabolic, pro and anti-inflammatory, pro-oxidant and antioxidant systems, among others.

Advances in technology and data acquisition have simplified the collection and storage of large datasets with long time series, finding increasingly frequent and varied fields of application, including biomedical and data mining areas. In this way, the process of evaluating large volumes of data is an invaluable process 
and the recent studies emphasize the use of AI methods with promising results [23] [24]. Supervised ANN methods address individual differences, rather than considering differences between groups, as do more traditional statistical comparisons, and classifying individuals in order to contribute to the clinical decision making process. These methods generate a model using a training set that includes input and output data. After the classification process, the model is tested using external test data to estimate the predictive capacity of the model. These methods are also sensitive to spatially distributed and subtle brain effects that would otherwise be indistinguishable by applying traditional univariate methods that focus on gross differences at the group level [23]. Although ANN methods are used in biomedical studies, AI techniques in psychiatric disorders are still incipient. Several neuroimaging studies of [34] use AI techniques and neural networks to look for possible changes in BD patients. In addition, these authors have described, from the clinical point of view, findings relevant to the pathophysiological understanding of bipolar disorder. In this sense, our study has demonstrated that there is an interaction between several neurochemical and inflammatory factors that may be directly involved in BP and SZ.

Regarding peripheral markers, there are still few studies that used AI techniques to identify biomarkers in patients with bipolar disorder or schizophrenia. A study by [35] was highlighted. The Space Vector Machine (SVM) algorithm differentiated patients with bipolar disorder from healthy controls with a predictive accuracy of $72.5 \%$, and patients with schizophrenia from healthy subjects with a prediction accuracy of $77.5 \%$. However, the algorithm was not able to differentiate patients with bipolar disorder from patients with schizophrenia (REF). In our study, although using a different technique, it found an accuracy of $92 \%$ when comparing patients with $\mathrm{BD}$ with healthy individuals, and $93 \%$ when we compared SZ with healthy individuals. Moreover, our findings differentiate patients with bipolar disorder from patients with schizophrenia; it was found an accuracy of $92 \%$. It is necessary to point out that in the study of [35]; the evaluations were carried out on blood samples, whereas the sample of this study was brain tissue.

This is a study to evaluate the feasibility of using a biomarker tool developed with ANN algorithms to identify a patient with bipolar disorder or schizophrenia when compared to healthy controls. However, the present work has some limitations: 1) Our sample was small as we used a brain from post-mortem tissue; 2) the majority of individuals were taking medication, a factor that influences the results obtained. Despite these limitations, future studies should assess larger samples from multiple centers; use advanced mathematical techniques combined with other biological and clinical variables to improve our knowledge about schizophrenia and bipolar disorder. Moreover, in the last years the use of ANNs has been growing as to a promise approach in basic and clinical studies. Here, our findings suggest that artificial neural network could be valid to detect the role of markers in the involvement of inflammatory mechanisms in the pathophysiology of bipolar disorder and schizophrenia. 


\section{Acknowledgements}

This study was supported by the following government agencies: Coordenação de Aperfeiçoamento de pessoal de Ensino Superior (CAPES) and Conselho Nacional de Desenvolvimento Científico e Tecnológico (CNPq). We would like to thank all participants of this study as well as all participating centers for the collaboration and for access to the samples. We gratefully acknowledge the Stanley Medical Research Institute for the data bank availability.

\section{Conflicts of Interest}

The authors declare no conflicts of interest regarding the publication of this paper.

\section{References}

[1] Mei, Y., Hontecillas, R., Zhang, X., Carbo, A. and Bassaganya-Riera, J. (2013) Neural Network Models for Classifying Immune Cell Subsets. 2013 IEEE International Conference on Bioinformatics and Biomedicine, Shanghai, 18-21 December 2013, 5-11. https://doi.org/10.1109/BIBM.2013.6732614

[2] Hassoun, M.H. (2009) Fundamentals of Artificial Neural Networks. The MIT Press, Cambridge.

[3] Amato, F., López, A., Peña-Méndez, E.M., Vaňhara, P., Hampl, A. and Havel, J. (2013) Artificial Neural Networks in Medical Diagnosis. Journal of Applied Biomedicine, 11, 47-58. https://doi.org/10.2478/v10136-012-0031-x

[4] Gant, V., Rodway, S. and Wyatt, J. (2001) Artificial Neural Networks: Practical Considerations for Clinical Application. Cambridge University Press, Cambridge, 329-356.

[5] Singh, P., Singh, S. and Pandi-Jain, G.S. (2018) Effective Heart Disease Prediction System Using Data Mining Techniques. International Journal of Nanomedicine, 13, 121-124. https://doi.org/10.2147/IJN.S124998

[6] Doan, N.T., et al. (2017) Distinct Multivariate Brain Morphological Patterns and their Added Predictive Value with Cognitive and Polygenic Risk Scores in Mental Disorders. NeuroImage: Clinical, 15, 719-731. https://doi.org/10.1016/j.nicl.2017.06.014

[7] Clemente, A.S., et al. (2015) Bipolar Disorder Prevalence: A Systematic Review and Meta-Analysis of the Literature. Brazilian Journal of Psychiatry, 37, 155-161. https://doi.org/10.1590/1516-4446-2012-1693

[8] Perälä, J., et al. (2007) Lifetime Prevalence of Psychotic and Bipolar I Disorders in a General Population. Archives of General Psychiatry, 64, 19-28. https://doi.org/10.1001/archpsyc.64.1.19

[9] Bahn, S. (2002) Gene Expression in Bipolar Disorder and Schizophrenia: New Approaches to Old Problems. Bipolar Disorders, 4, 70-72. https://doi.org/10.1034/j.1399-5618.4.s1.28.x

[10] American Psychiatric Association (2017) Diagnostic and Statistical Manual of Mental Disorders: DSM-5. CBS Publishers \& Distributors, Pvt. Ltd., Delhi.

[11] Jansen, K., Magalhães, P.V., Pinheiro, R.T., Kapczinski, F. and Silva, R.A.D. (2012) Early Functional Impairment in Bipolar Youth: A Nested Population-Based Case-Control Study. Journal of Affective Disorders, 142, 208-212. 
https://doi.org/10.1016/j.jad.2012.04.028

[12] Wells, R., Kite, B., Breckenridge, E. and Sunbury, T. (2018) Community Mental Health Center Integrated Care Outcomes. Psychiatric Quarterly, 89, 969-982. https://doi.org/10.1007/s11126-018-9594-3

[13] Lotan, A., et al. (2014) Neuroinformatic Analyses of Common and Distinct Genetic Components Associated with Major Neuropsychiatric Disorders. Frontiers in Neuroscience, 8, 331 .

[14] Post, R.M. and Kalivas, P. (2013) Bipolar Disorder and Substance Misuse: Pathological and Therapeutic Implications of their Comorbidity and Cross-Sensitisation. British Journal of Psychiatry, 202, 172-176. https://doi.org/10.1192/bjp.bp.112.116855

[15] Munkholm, K., Weikop, P., Kessing, L.V. and Vinberg, M. (2015) Elevated Levels of IL-6 and IL-18 in Manic and Hypomanic States in Rapid Cycling Bipolar Disorder Patients. Brain, Behavior, and Immunity, 43, 205-213. https://doi.org/10.1016/j.bbi.2014.09.021

[16] Ghafelehbashi, H., Kakhki, M.P., Kular, L., Moghbelinejad, S. and Ghafelehbashi, S.H. (2017) Decreased Expression of IFNG-AS1, IFNG and IL-1B Inflammatory Genes in Medicated Schizophrenia and Bipolar Patients. Scandinavian Journal of Immunology, 86, 479-485. https://doi.org/10.1111/sji.12620

[17] Bauer, I.E., Pascoe, M.C., Wollenhaupt-Aguiar, B., Kapczinski, F. and Soares, J.C. (2014) Inflammatory Mediators of Cognitive Impairment in Bipolar Disorder. Journal of Psychiatric Research, 56, 18-27. https://doi.org/10.1016/j.jpsychires.2014.04.017

[18] Prata, J., Santos, S.G., Almeida, M.I., Coelho, R. and Barbosa, M.A. (2017) Bridging Autism Spectrum Disorders and Schizophrenia through Inflammation and Biomarkers Pre-Clinical and Clinical Investigations. Journal of Neuroinflammation, 14, 179. https://doi.org/10.1186/s12974-017-0938-y

[19] Luo, Y., He, H., Zhang, M., Huang, X. and Fan, N. (2016) Altered Serum Levels of TNF- $\alpha$, IL-6 and IL-18 in Manic, Depressive, Mixed State of Bipolar Disorder Patients. Psychiatry Research, 244, 19-23. https://doi.org/10.1016/j.psychres.2016.07.027

[20] Dembic, Z. (2015) The Cytokines of the Immune System: The Role of Cytokines in Disease Related to Immune Response. Elsevier/Academic Press, Cambridge.

[21] Moreira, F.P., et al. (2018) Childhood Trauma and Increased Peripheral Cytokines in Young Adults with Major Depressive: Population-Based Study. Journal of Neuroimmunology, 319, 112-116. https://doi.org/10.1016/j.jneuroim.2018.02.018

[22] Aakerlund, L.P. (2000) Artificial Neural Networks and Schizophrenia. Nordic Journal of Psychiatry, 54, 221-224. https://doi.org/10.1080/080394800750019141

[23] Erguzel, T.T., Tas, C. and Cebi, M.A. (2015) Wrapper-Based Approach for Feature Selection and Classification of Major Depressive Disorder. Bipolar Disorders. Computers in Biology and Medicine, 64, 127-137.

[24] Passos, I.C., et al. (2016) Identifying a Clinical Signature of Suicidality among Patients with Mood Disorders: A Pilot Study Using a Machine Learning Approach. Journal of Affective Disorders, 193, 109-116. https://doi.org/10.1016/j.jad.2015.12.066

[25] Torrey, E., Webster, M., Knable, M., Johnston, N. and Yolken, R.H. (2000) The Stanley Foundation Brain Collection and Neuropathology Consortium. Schizophrenia Research, 44, 151-155. https://doi.org/10.1016/S0920-9964(99)00192-9 
[26] Array Collection. The Stanley Neuropathology Consortium Integrative Database. http://sncid.stanleyresearch.org/Default.aspx?ReturnUrl=\%2fData\%2fArrayCollecti onData.aspx

[27] Kim, S. and Webster, M.J. (2010) The Stanley Neuropathology Consortium Integrative Database: A Novel, Web-Based Tool for Exploring Neuropathological Markers in Psychiatric Disorders and the Biological Processes Associated with Abnormalities of Those Markers. Neuropsychopharmacology, Nature Publishing Group, 35, 473-482. http://www.ncbi.nlm.nih.gov/pmc/articles/PMC3055386/ https://doi.org/10.1038/npp.2009.151

[28] Harris, L.W., et al. (2012) Comparison of Peripheral and Central Schizophrenia Biomarker Profiles. PLoS ONE, 7, e46368. https://doi.org/10.1371/journal.pone.0046368

[29] Luca, M.D., et al. (2005) Artificial Neural Networks Allow the Use of Simultaneous Measurements of Alzheimer Disease Markers for Early Detection of the Disease. Journal of Translational Medicine, 3, 30. https://doi.org/10.1186/1479-5876-3-30

[30] OpenNN. Open Neural Networks Library. http://www.opennn.net/

[31] Serretti, A., Zanardi, R., Mandelli, L., Smeraldi, E. and Colombo, C. (2007) A Neural Network Model for Combining Clinical Predictors of Antidepressant Response in Mood Disorders. Journal of Affective Disorders, 98, 239-245. https://doi.org/10.1016/j.jad.2006.08.008

[32] Metin, S.Z., et al. (2017) The Use of Quantitative EEG for Differentiating Frontotemporal Dementia From Late-Onset Bipolar Disorder. Clinical EEG and Neuros cience, 49, 171-176. https://doi.org/10.1177/1550059417750914

[33] Neuropathology Consortium Data Stanley Research Neuropathology Consortium. http://www.stanleyresearch.org/brain-research/neuropathology-consortium

[34] Mon-Ju, W., et al. (2017) Identification and Individualized Prediction of Clinical Phenotypes in Bipolar Disorders Using Neurocognitive Data, Neuroimage Scans and Machine Learning. Neuroimage, 145, 254-264. https://doi.org/10.1016/j.neuroimage.2016.02.016

[35] Pinto, J.V., et al. (2017) Peripheral Biomarker Signatures of Bipolar Disorder and Schizophrenia: A Machine Learning Approach. Schizophrenia Research, 188, 182-184. https://doi.org/10.1016/j.schres.2017.01.018 